\title{
Formación en red de habilidades de investigación en la Universidad Estatal a Distancia de Costa Rica
}

\author{
Andrés Segura-Castillo y Silvia Vázquez Olguín \\ Universidad Estatal a Distancia, 474-2050 San Pedro Montes de Oca, San José, Costa Rica; asegurac@uned.ac.cr; silvia.vazquezolguin@gmail.com
}

Recibido 30-XI-2011 Corregido 3-II-2012 Aceptado 14-III-2012

\begin{abstract}
Development of e-Learning based research skills at the Costa Rican Distance Education University. The Costa Rican Distance Education University has created the Proto-Red de Centros Universitarios para la Investigación which consists of a virtual social network aimed at developing research skills among its students. Social Network Analysis methods were applied to 174 articles produced over a year in this network and the corresponding results show, besides an unexpected user profile, the existence of incipient virtual communities based on common interest topics. Furthermore, users with outstanding performance as information providers were identified. As a result of these findings, promoters of the network are suggested to strengthen this initiative through activities that take advantage of the identified profile. There is a need for e-Learning based development of research skills, a model that guides user activities and makes the most of the interests found on the virtual communities. Social Networks Analysis is an effective tool for the identification of distinguished users in virtual social networks.
\end{abstract}

\section{KEY WORDS}

Social networks, skills, research, distance education.

\section{RESUMEN}

La Universidad Estatal a Distancia de Costa Rica incursionó en la formación en red de habilidades de investigación en sus estudiantes mediante una red social virtual denominada Proto-Red de Centros Universitarios para la Investigación. Este artículo presenta los resultados de un Análisis de Redes Sociales de 174 artículos producidos por la red durante un año. Se halló un perfil inesperado del usuario, el surgimiento de comunidades virtuales alrededor de temas de interés común y la existencia de usuarios que sobresalen como mediadores del flujo de información. Se sugiere la intervención por parte de los promotores de esta iniciativa mediante actividades focalizadas en los centros universitarios que fortalezcan la red y aprovechen las características reflejadas en el perfil encontrado. A su vez se enfatiza la necesidad de un modelo de formación en red que guíe el quehacer de los miembros, de forma tal que se aprovechen los espacios creados por las comunidades virtuales. Se resalta además que el uso de métodos Análisis de Redes Sociales es una estrategia adecuada para la generación de indicadores para la identificación usuarios sobresalientes espacios sociales virtuales.

\section{PALABRAS CLAVE}

Redes sociales, habilidades, investigación, educación a distancia.
El estudio de la dinámica de las relaciones sociales mediante técnicas cuantitativas tiene su origen en la década de los treinta del siglo veinte con la aparición de herramientas como los sociogramas, las matrices sociales, la teoría de grafos y otras, que permiten medir la interacción social (Wasserman et al. 2005). Progresivamente, este tipo de técnicas evolucionaron en complejidad y su uso aumentó hasta derivar, a mediados de los años setenta del período mencionado, en el Análisis de Redes Sociales (ARS). Este campo es hoy en día una rama consolidada entre las ciencias sociales y cuenta con teorías, modelos, metodología y aplicaciones propias. Su nombre se debe al hecho de que los vínculos sociales son entendidos como un tejido de relaciones y procesos entre entidades, es decir, como redes sociales (Wasserman \& Faust 1994). Con respecto a la naturaleza de las redes sociales, Wasserman \& Faust (1994) señalan que las siguientes características son básicas:

- Una interdependencia entre los entes que forman parte de la red.

- Un flujo de recursos (materiales y no materiales) a través de los enlaces que unen a las entidades en la red. 
- La influencia de la red misma en las oportunidades y/o restricciones para la acción individual de sus integrantes.

Recientemente las redes sociales propiciadas por el uso de tecnologías de información han beneficiado considerablemente al ARS, especialmente por la aparición de espacios en Internet, que permiten la interacción de múltiples usuarios y la conformación de grupos en torno a temas de interés común. El punto quizá más interesante de esta situación es que el comportamiento de estos empata perfectamente con los postulados del ARS y es un campo de estudio fértil donde aplicar los métodos de la disciplina.

La Universidad Estatal a Distancia de Costa Rica (UNED) no es ajena a este tipo de procesos, por el contrario, históricamente su modelo de enseñanza a distancia se ha constituido a partir de la mediación tecnológica y de la interacción de sus usuarios mediante una red de información (UNED 2009). La UNED misma puede concebirse como una compleja red social, compuesta a su vez de múltiples redes sociales. Una de ellas es la Proto-Red de Centros Universitarios para la Investigación (Proto-Red) promovida por la Vicerrectoría de Investigación de la institución, la cual consiste en un espacio virtual a disposición de sus estudiantes y funcionarios, con el objetivo de impulsar una cultura que según Chinchilla et al. (2010) propicie:

1. Una dinámica de trabajo colaborativo en red, basada en el intercambio de información, y el establecimiento de relaciones entre los centros universitarios interesados en desarrollar, promocionar y fortalecer la investigación.

2. Procesos de formación que potencien las capacidades para la investigación de los centros universitarios de acuerdo con el contexto específico de cada uno de ellos.

Cabe aclarar que en la actualidad las instituciones de educación superior buscan formar profesionales con sólidas bases para la investigación mediante métodos cada vez más participativos, ya que, independientemente de si el estudiante desea dedicarse exclusivamente a investigar o no, la dinámica de la sociedad actual demanda un constante seguimiento y una clara comprensión del nuevo conocimiento científico. Asimismo la vida laboral de hoy en día requiere, en muchas ocasiones, la incorporación o la adaptación de resultados de investigaciones al quehacer laboral, así como el uso de herramientas de naturaleza científica (publicaciones, métodos, entre otros) en muchos de sus ámbitos. Además, la capacidad de innovar, es decir, producir nuevo conocimiento es, sin lugar a dudas, un valor agregado en cualquier profesión, por lo que la formación de habilidades para la investigación que potencien en los estudiantes universitarios dicha capacidad, es una pieza fundamental para la innovación en nuestras sociedades (Murtonen et al. 2008).

Ahora bien, es necesario aludir al hecho de que en el contexto de la educación a distancia la formación de habilidades para investigación en estudiantes no es una tarea trivial. La ausencia de un guía o profesor en el sentido tradicional de la educación superior repercute, al menos en la UNED, en las posibilidades que el alumno tiene para vincularse a procesos de investigación (Chinchilla et al. 2010). Dada dicha situación, una posibilidad que emerge para la UNED es la formación en red, es decir, el uso de entornos virtuales como propulsores de cambios educativos sustentados en nuevas dinámicas de interacción y por ende como medio para el aprendizaje de nuevas capacidades (Suárez 2010). La opción resulta adecuada para la UNED, en primera instancia, por la actitud favorable que demuestran en general los estudiantes universitarios hacia este tipo de ambientes virtuales (Espuny et al. 2011) y además por la reciente tendencia de entender la formación en red, como un objeto de estudio vital para la elaboración de nuevos paradigmas de educación acordes con las necesidades de la sociedad actual (Suárez 2010).

De esta forma, la UNED apuesta por Proto-Red como un espacio de formación en red que propicia, tal y como sus objetivos aclaran, una cultura en el estudiante, que incentiva el intercambio de información y el desarrollo de habilidades para investigar. Proto-Red resulta así, una alternativa innovadora en el ámbito de la educación a distancia y su análisis puede contribuir a la incorporación sistemática de la formación en red en los procesos educativos de la UNED y al establecimiento de un modelo de trabajo que maximice la posibilidad de formar jóvenes investigadores en un ambiente de enseñanza a distancia. El estudio muestra cuantitativamente cómo dicha cultura se manifiesta en la configuración de esta red, en su dinámica en el espacio virtual así como en los roles que emergen, partiendo de la sistematización y la categorización de las publicaciones, visitas y comentarios de los usuarios que pertenecen a Proto-Red. Además se discute, dados los resultados obtenidos mediante el uso de métodos de análisis de redes sociales, su eventual evolución y las posibles medidas a tomar para potenciar su impacto en la UNED y en general en la educación a distancia. Cabe resaltar que Proto-Red puede ser accedida en el siguiente enlace en Internet: http://investiga.uned.ac.cr/protored. 


\section{METODOLOGÍA}

El enfoque metodológico empleado para el estudio de Proto-Red es en este caso cuantitativo, con la finalidad de obtener métricas que permitan inferir la dinámica de la red. Dada la cantidad y distribución geográfica de los usuarios, una aproximación cualitativa más cercana, que captara las opiniones detalladas de los participantes, con entrevistas y actividades presenciales por ejemplo, no era viable en el momento de inicio de la investigación, aunque no se descarta la posibilidad de estudiar cualitativamente el fenómeno posteriormente. Además, dado que la Proto-Red se manifiesta, esencialmente, en un medio virtual, se prefirió el uso de las herramientas que la misma red social brinda para extraer los datos que evidencian su dinámica y que serán analizados posteriormente.

Es importante mencionar que se parte de la existencia de un perfil particular en la población usuaria de ProtoRed con las siguientes características:

- Mayoritariamente masculina.

- Eminentemente rural.

- Limitado acceso a internet y escasa tenencia de equipo computacional en sus hogares.

Esta serie de presunciones surge de las características de la población estudiantil que se supone atiende la UNED (UNED 2009), así como de los indicadores costarricenses de acceso a las tecnologías de información (INEC 2010) que se le atribuyen a las localidades de los centros universitarios participantes de la Proto-Red, mayoritariamente ubicados en zonas alejadas del área metropolitana de Costa Rica. El perfil planteado es en sí una hipótesis necesaria para la posterior categorización de los usuarios de la red. Dicha clasificación será a su vez, un insumo importante para elegir los métodos de ARS a utilizar. Además se asume que es posible determinar la configuración y las dinámicas de la Proto-Red, a partir de la información obtenida del registro de la participación (visitas, publicaciones y comentarios) de los miembros de la red. Cabe aclarar que el sitio en Internet de la Proto-Red cuenta con un administrador de contenidos capaz de proveer todos los datos necesarios.

A partir de los supuestos mencionados se plantea los siguientes pasos metodológicos para estudiar a Proto-Red desde el ARS:

- Definición de las variables de análisis.

- Categorización de los usuarios de la red.

- Sistematización de la participación en el sitio.

- Visualización y evaluación mediante ARS.

A continuación se describe en detalle cada uno de ellos.

\section{Definición de las variables de análisis}

Las variables de análisis elegidas surgen, en primera instancia, de las características demográficas y de acceso a las tecnologías de información de los usuarios de Proto-Red. Además, siguiendo una metodología similar a la de Ulrike \& Panayiotis (2009), las visitas, publicaciones y comentarios de los usuarios guían la escogencia de otras variables que miden la participación en la red sin imponer criterios desde los investigadores.

Así, los aspectos analizados en los usuarios son los siguientes:

- Sexo: Identifica si el usuario es hombre o mujer.

- Condición académica: Señala si el usuario es estudiante o funcionario del centro universitario.

- Centro universitario: Centro universitario al cual pertenece el usuario.

- Acceso Internet: Muestra si cuenta con algún medio de acceso a Internet.

Cabe aclarar que dichas variables coinciden también con las características del perfil supuesto para la población usuaria de Proto-Red, con el fin de aceptar o descartar nuestra hipótesis.

Asimismo, la participación de los miembros de la red fue estudiada según el Centro Universitario de pertenencia, lugar donde se gesta el vínculo del usuario con ProtoRed. En este sentido se tomaron en cuenta las siguientes variables:

- Cantidad de publicaciones por Centro Universitario.

- Cantidad de visitas por Centro Universitario.

- Cantidad de comentarios por Centro Universitario.

Cabe aclarar que también se incluyó a la Vicerrectoría de Investigación como si fuera un centro universitario, ya que Proto-Red es promovida en la UNED por esta instancia y existen usuarios de la red que pertenecen a dicha vicerrectoría. De esta forma es posible obtener una categorización de la población usuaria en dos niveles, a saber, el individual y el grupal.

\section{Categorización de los usuarios de la red}

La categorización de los usuarios de Proto-Red parte de dos puntos, a saber:

- La hipótesis hecha en relación al perfil de la población usuaria de Proto-Red.

- Las variables de análisis elegidas en el paso metodológico anterior. 
Con el fin de confrontar el perfil supuesto y obtener una categorización objetiva se elaboró una encuesta digital entre los participantes de Proto-Red. Las cifras obtenidas a partir de dicho instrumento son presentadas en la posterior sección de resultados. Este paso corresponde a la caracterización individual de los miembros de la red.

Seguidamente el nivel grupal del perfil se depuró mediante una sistematización de la participación de los usuarios, paso que es aclarado a continuación.

\section{Sistematización de la participación en el sitio}

En la actualidad es común caracterizar a las redes sociales virtuales según el tráfico de información que generan sus usuarios en los respectivos sitios en Internet, por ejemplo, mediante la cantidad de visitas en un período dado, el tipo de mensajes que envían sus usuarios, el contenido de dichos mensajes, los vínculos que establecen los usuarios, entre otros. Redes como Facebook y Flickr, ya han sido observadas mediante este tipo de metodología y su estudio ha evidenciado la existencia de estructuras sociales similares a las que acontecen en grupos presenciales (Nazir et al. 2008), y además otras características propias del ambiente virtual que no suceden en la cotidianeidad (Cha et al. 2009).

En nuestro caso, la sistematización de la participación en la red parte de la cuantificación y categorización de los resultados obtenidos mediante el seguimiento de las variables elegidas previamente: las publicaciones, las visitas y los comentarios, tanto por centro universitario como a nivel individual. Los datos obtenidos en este punto permiten profundizar en el análisis y establecer intereses y áreas temáticas en la red que colaboran a establecer un perfil más detallado de los usuarios (Ulrike \& Panayiotis 2009). Cabe aclarar que el período estudiado inicia a principios el segundo semestre del 2010 y finalizó al término del primer semestre del 2011.

También es importante señalar que los resultados de este paso metodológico guían la elección de los métodos de ARS utilizados para evaluar la configuración de ProtoRed. El producto de la sistematización se muestra más adelante en la sección de resultados.

\section{Visualización y evaluación mediante ARS}

La visualización de datos ha sido de vital importancia para el ARS. Desde sus inicios, la representación de la red y sus componentes en forma de nodos interconectados mediante enlaces, asume que la red tiene propiedades que se ven reflejadas en el locus adquirido por los usuarios participantes y en el flujo de la información (Brandes et al.
2005). Asimismo es importante recordar que la inquietud fundamental del ARS es establecer una serie de métricas que describan y expliquen el comportamiento de las redes sociales. Cabe resaltar, que dicho análisis asume que las redes poseen una estructura, que se hace evidente en los patrones regulares de interacción entre las entidades concretas (personas, grupos pequeños, organizaciones, entre otros) que participan en ellas (Knoke \& Yang 2008).

En nuestro caso es posible entonces visualizar la red con sus respectivos nodos y enlaces a partir del flujo de información existente entre las publicaciones de Proto-Red y los comentarios entre los usuarios. Cabe aclarar que el perfil obtenido en el paso anterior, tanto a nivel individual como grupal, brinda los datos necesarios para evidenciar dicho tráfico y guía los pasos a seguir a partir de este punto. Así, inicialmente se establece una formalización para Proto-Red de la siguiente manera:

- Sea $R$ una red social tal que: $A \in R$ sii publica $(A, X) \vee c o-$ menta $(A, X)$, es decir, el usuario $A$ pertenece a la red social $R$ si y sólo si publica o comenta un artículo $X$.

- Además existe un enlace entre cualesquiera dos usuarios de la red si se cumple la siguiente condición: enlace $(A, B) \rightarrow$ publica $(A, X) \wedge$ comenta $(B, X)$, es decir, el enlace entre los usuarios $A$ y $B$ se evidencia porque $A$ publica el artículo $X$ y $B$ comenta el artículo $X$.

Dada dicha formalización es posible visualizar la red con usuarios enlazados por los artículos y los comentarios generados. Con el objetivo de facilitar la graficación de la red, se utilizó el software de análisis de redes sociales AGNA (Agna Project 2010). La visualización resultante muestra la estructura de la red en términos del flujo de información.

Ahora bien el ARS tiene la particularidad de contar con métricas que complementan la visualización y que permiten dilucidar, entre otras características, los roles que asumen algunos usuarios durante su participación de la red, así como la complejidad de las interacciones. Con el objetivo de ampliar el análisis y la discusión, la investigación midió además el grado de intermediación de todos los usuarios (Knoke \& Yang 2008). Los resultados correspondientes están incluidos en la siguiente sección.

\section{RESULTADOS}

\section{Categorización de los usuarios de la red}

Tal y como se mencionó anteriormente, para afinar el perfil supuesto inicialmente se elaboró una encuesta digital, la cual se denominó "¿Quiénes son nuestros usuarios?", aplicada al inicio del período de estudio. El instrumento fue 
respondido vía Proto-Red por 20 usuarios activos en ese entonces, cantidad correspondiente a un $57 \%$ de las y los usuarios que participaban en ese momento de la iniciativa. Cabe resaltar que ningún Centro Universitario participante de la red se quedó sin representación en la encuesta, razón por la cual consideramos que los resultados son relevantes para sustentar o descartar las hipótesis hechas al inicio y por ende afinar el perfil de la población usuaria.

En relación a la variable sexo se obtuvo que la población es $60 \%$ femenina. Además los resultados indican una mayoría de participación estudiantil con un $55 \%$. Asimismo el acceso a Internet entre los miembros de la ProtoRed se da primordialmente en el centro universitario con un $40 \%$, seguido de equipo de cómputo en su propia casa con un $35 \%$, otros medios (teléfono celular básicamente) con un $20 \%$ y un $5 \%$ en cafés internet. Es importante resaltar que ningún miembro reportó la ausencia de acceso a Internet. El instrumento demostró además que el porcentaje de participación de los usuarios de la red se distribuía por centro universitario como se observa en la Figura 1.

Además los datos relacionados con la actividad grupal de los usuarios, particularmente, la cantidad de publicaciones muestran los siguientes resultados (Fig. 2), donde el grupo de la Vicerrectoría de Investigación de la UNED es el que acumula mayor cantidad de ellas.
Por otra parte, la Figura 3 denota la distribución de menor a mayor de la cantidad de visitas a los artículos publicados por cada uno de los grupos. Las publicaciones más visitadas fueron producidas igualmente por el grupo de la Vicerrectoría de Investigación de la UNED. Es de vital importancia aclarar que el conteo de visitas mostrado ya ha sido filtrado, es decir, no contabiliza múltiples visitas del mismo usuario a la misma página ni visitas generadas automáticamente por programas de indización tipo Google.

Finalmente la distribución de la totalidad de comentarios generados a partir de las publicaciones hechas por los grupos es la siguiente, donde el grupo de la Vicerrectoría de Investigación de la UNED recibe el mayor número de comentarios (Fig. 4)

Los resultados presentados hasta el momento están relacionados con las variables establecidas para la caracterización de la población usuaria de la Proto-Red. Los resultados obtenidos permiten a su vez sistematizar el fenómeno de estudio a un nivel de profundidad mayor. A continuación se presenta el producto obtenido de dicha sistematización.

\section{Sistematización de la participación en el sitio}

La participación por parte de los usuarios ha sido denotada hasta el momento por la cantidad de artículos que

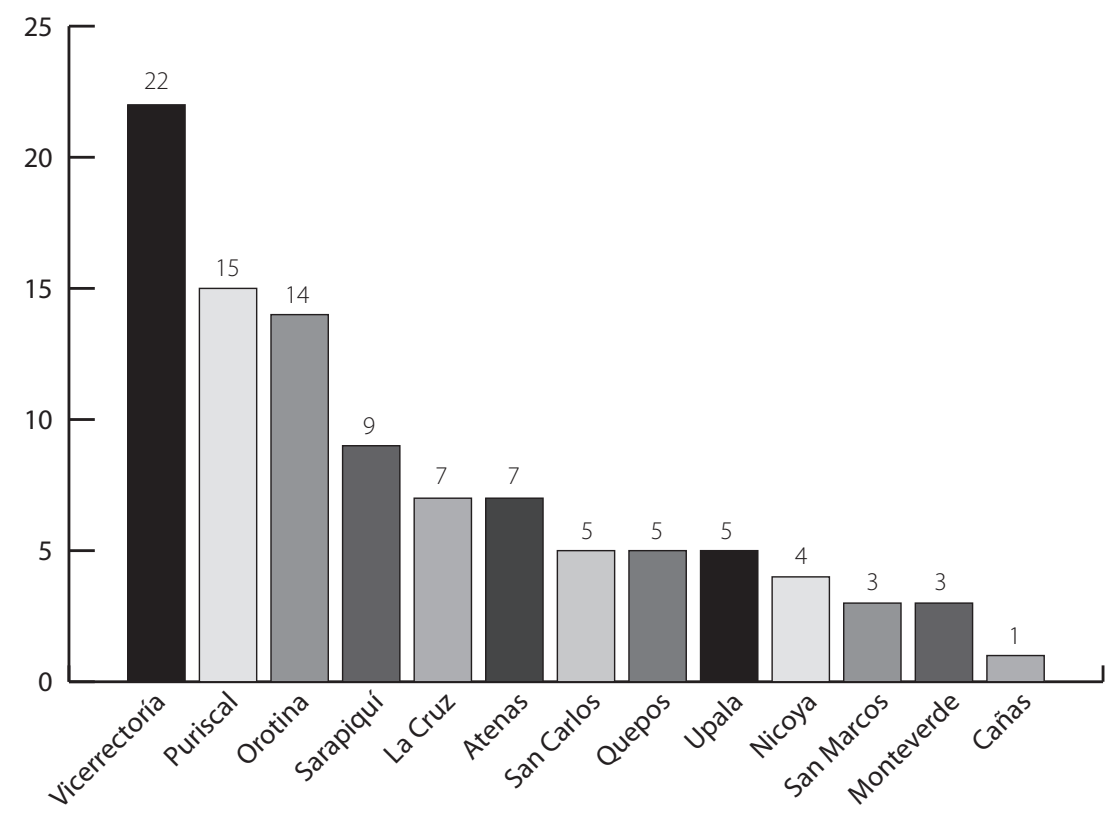

FIG.1. Porcentaje de participantes de Proto-Red según centro universitario. 


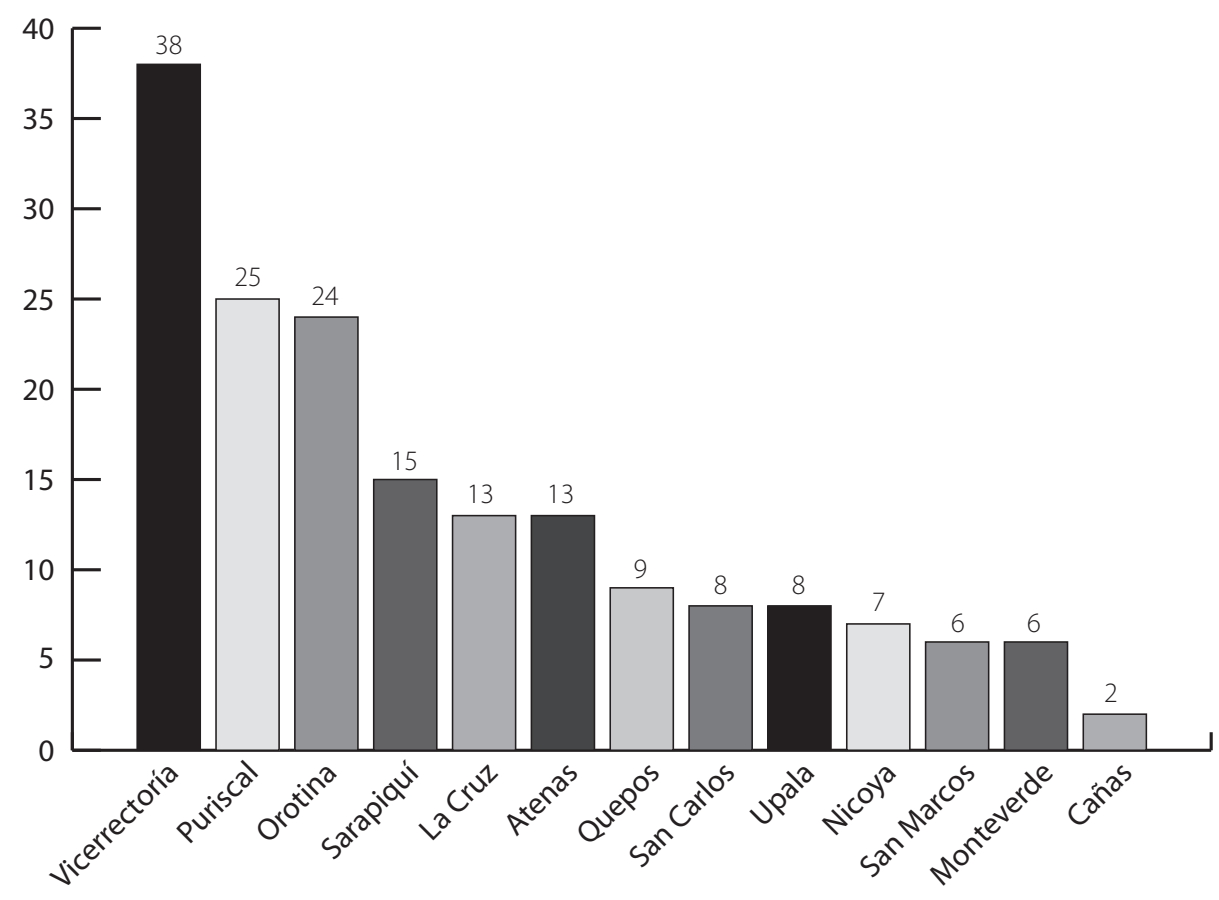

FIG. 2. Cantidad de publicaciones de Proto-Red por grupo.

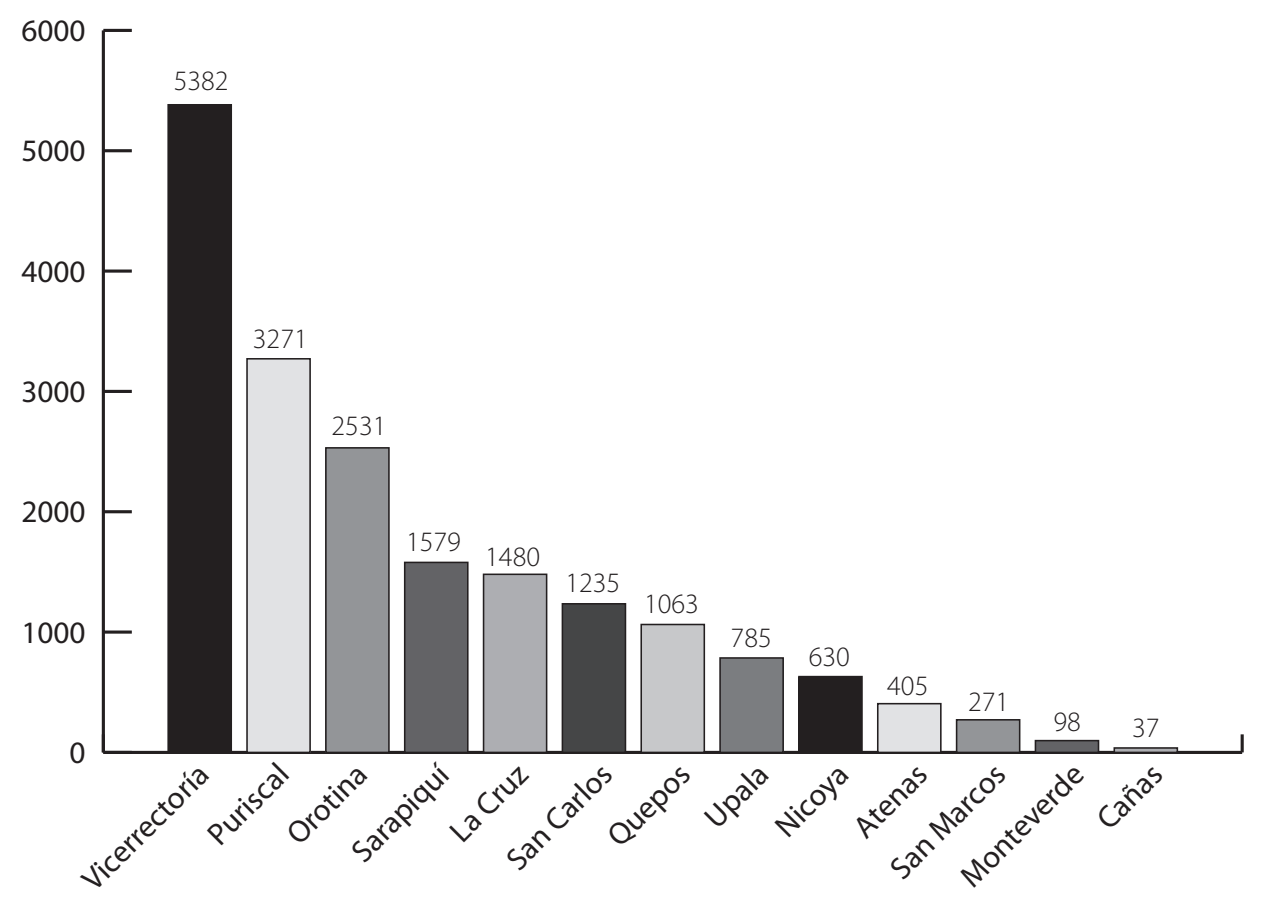

FIG. 3. Cantidad de visitas a las publicaciones de Proto-Red según grupo. 


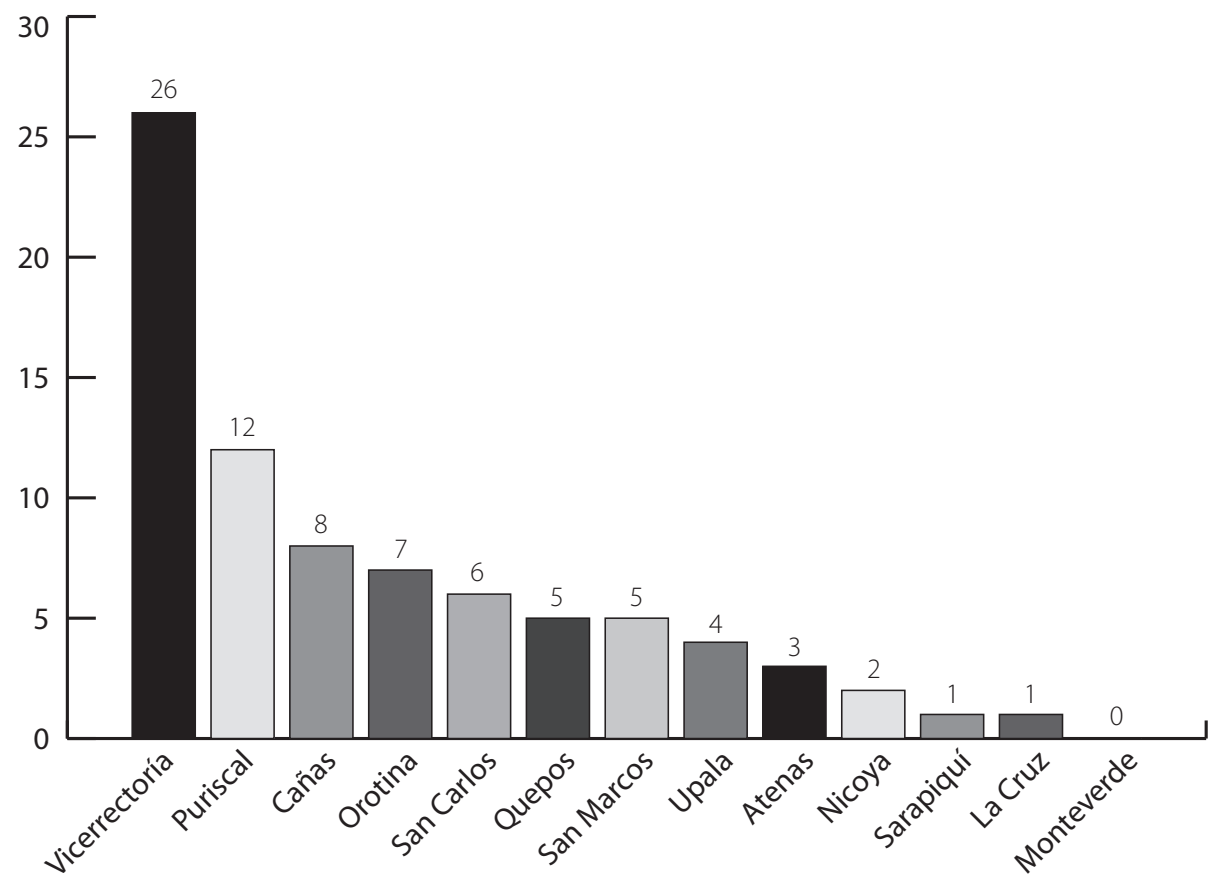

FIG. 4. Cantidad de comentarios a las publicaciones de Proto-Red según grupo.

publican, la cantidad de visitas realizadas a las publicaciones y sus comentarios. Ahora bien, de forma similar a la aproximación empleada por Li et al. (2008), en el caso de Proto-Red fue posible sistematizar las publicaciones y visitas según intereses particulares en los usuarios y categorizarlas a partir de ellos. Estos intereses son denotados por los miembros de la red tanto en el contenido de los artículos o comentarios, como en la clasificación utilizada por ellos para organizar el contenido de sus publicaciones. Así las categorías resultantes fueron:

- Investigación: Identifica un interés de los usuarios por participar de dinámicas de investigación y formación de habilidades en este sentido.

- Centros Universitarios (CUs): Artículos y visitas relacionadas con temas asociados a dinámicas muy propias de los centros, como horarios, fechas de matricula, servicios, entre otros.

- Comunidad: Esta categoría abarca temas comunitarios de interés de los participantes de la Proto-Red, por ejemplo festividades, problemáticas especificas de la comunidad, entre otras.
- Vida Estudiantil: Su contenido refleja las dinámicas extracurriculares experimentadas en los centros universitarios, por ejemplo, grupos de danza, deportes, entre otros.

- Proto-Red: Abarca temas relacionados con dinámicas vinculadas a la Proto-Red propiamente dicha, como giras, capacitaciones, talleres, entre otras.

- Temas ecológicos: Otra categoría que refleja un particular interés de un grupo de usuarios por el tema ambiental.

El gráfico de la figura 5 muestra la distribución de las publicaciones de la Proto-Red según las categorías obtenidas.

Además podemos visualizar las visitas distribuidas según las categorías en la figura 6.

Seguidamente mostramos el último nivel de análisis realizado y los resultados obtenidos con métodos de ARS a partir de los datos extraídos de Proto-Red. 


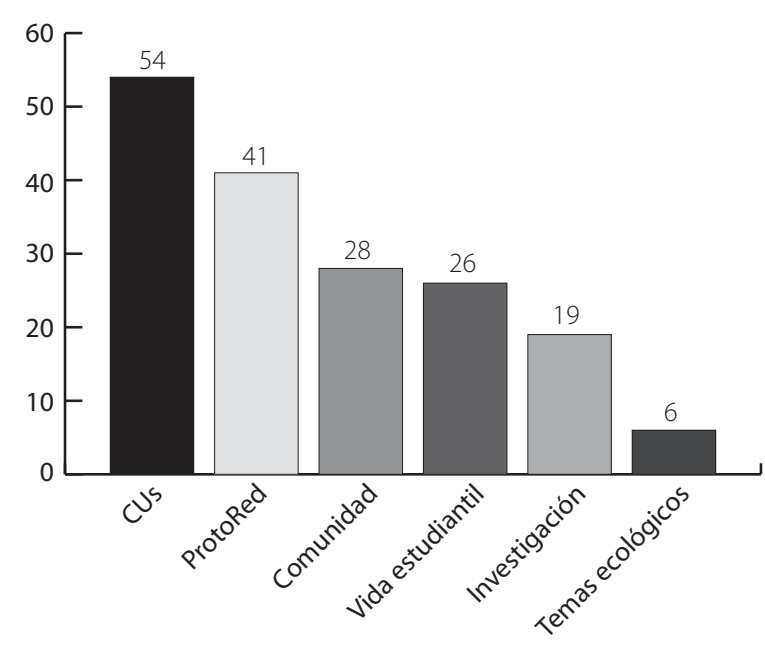

FIG. 5. Cantidad de publicaciones de la Proto-Red según categoría

\section{Visualización y evaluación de la red}

La figura 7 muestra la red obtenida según la formalización hecha en la anterior sección de metodología. Es importante mencionar que tanto usuarios como artículos se encuentran codificados para respetar su privacidad. Además la visualización de la red muestra únicamente artículos que han recibido comentarios, con el objetivo de no saturar el gráfico con información innecesaria.

Finalmente se calculó el grado de intermediación (Knoke \& Yang 2008) para cada uno de los usuarios visualizados. Se presenta los resultados de los cinco usuarios que lideran en este sentido en la figura 8.

En este punto es importante señalar que los usuarios con mayor grado de intermediación pertenecen a grupos que han mostrado la mayor actividad en publicaciones $y$ visitas. El siguiente apartado discute los resultados obtenidos y presenta posibles estrategias para la fortalecimiento de la red y el cumplimiento de sus objetivos.

\section{DISCUSIÓN}

Los resultados obtenidos durante la categorización de los usuarios permiten confrontar la hipótesis hecha en relación al perfil de la población usuaria de Proto-Red. Los datos generados a partir del instrumento " ¿Quiénes son nuestros usuarios?" indican que, contrario a la hipótesis, se cuenta con una población mayoritariamente femenina,

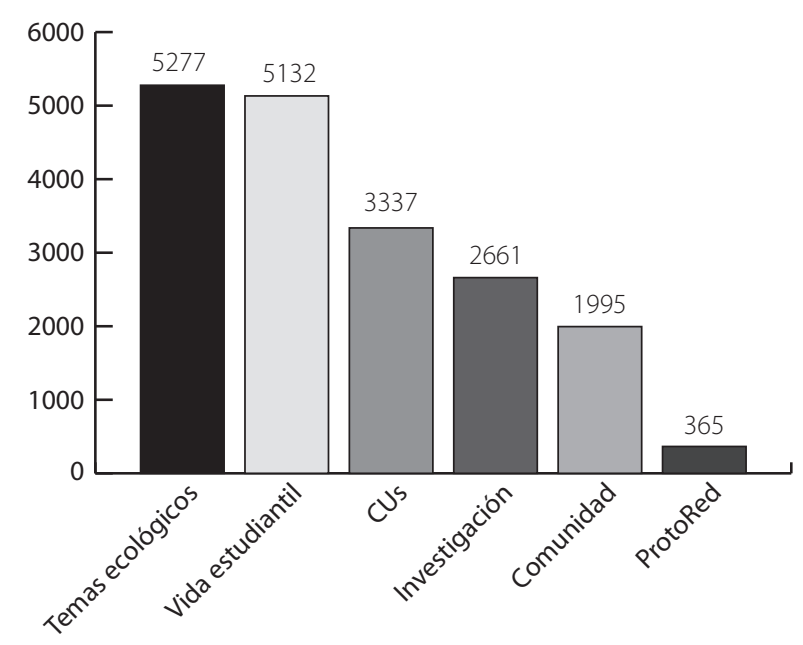

FIG. 6. Cantidad de visitas de la Proto-Red según categoría.

existe un alto grado de participación estudiantil, y los miembros de la red acceden sin mayor problema a Internet, principalmente acudiendo al centro universitario. Este perfil encontrado entre los miembros de la red sugiere que a pesar de las limitaciones normalmente atribuidas en términos de disponibilidad de las tecnologías de información en la población rural o alejada del área metropolitana, la existencia de centros universitarios en la UNED facilita el acceso a las mismas. Dicha situación plantea una interrogante muy importante para los promotores de la red, a saber, cómo aumentar la cantidad de usuarios aprovechando las condiciones brindadas en los centros, y desde estos puntos inculcar la formación en red. Es recomendable diseñar una estrategia que contemple actividades focalizadas en los centros, que induzcan a una mayor cantidad de posibles miembros a vincularse con la iniciativa. Un aumento en la población sería sin duda beneficioso para el impacto que la red pueda tener en el modelo de educación a distancia de la UNED.

Además es importante notar que los participantes de la red consultan mucho más de lo que publican o comentan artículos. Creemos que, de manera similar al caso reportado por Lampe et al. (2011) donde el uso de la red social Facebook muestra ser un elemento importante para las actividades estudiantiles, la Proto-Red solventa una necesidad de información y comunicación que la UNED no ha provisto en sus medios tradicionales. La posibilidad de encontrar información relevante para los temas de interés estudiantil en un ambiente virtual parece ser un elemento 


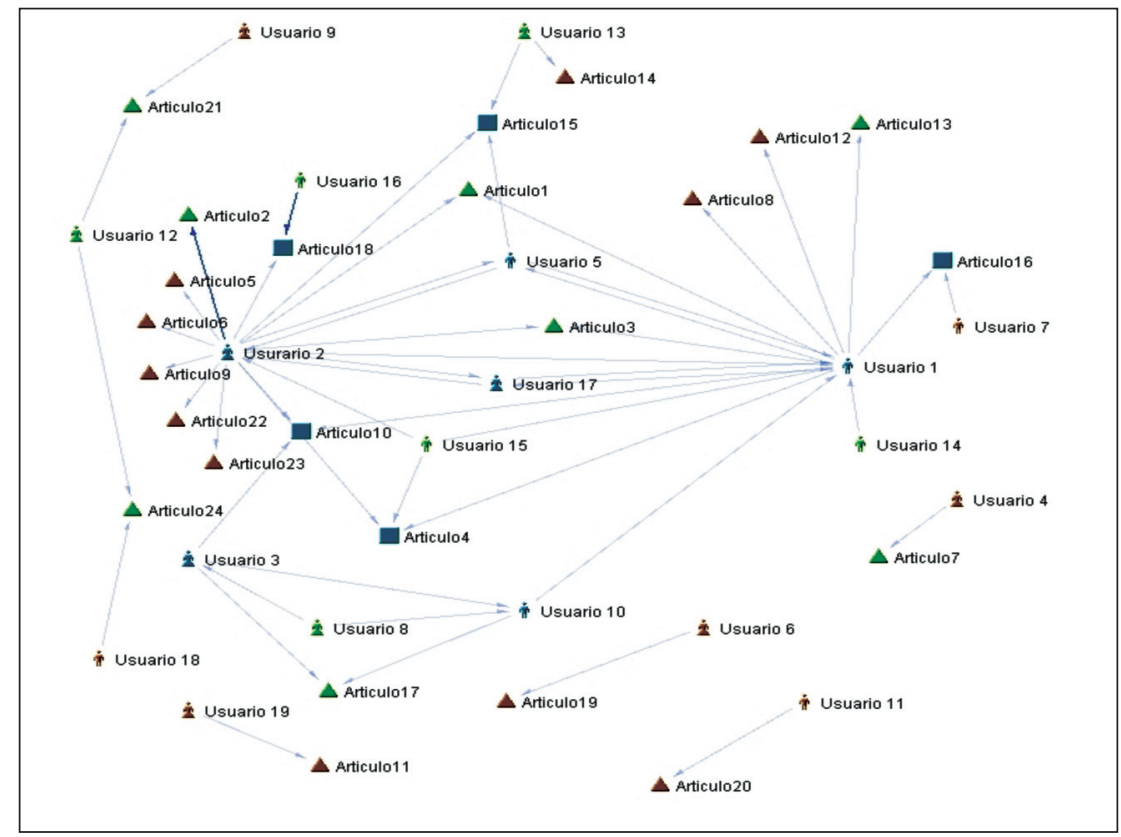

FIG. 7. Red obtenida a partir de artículos y comentarios.

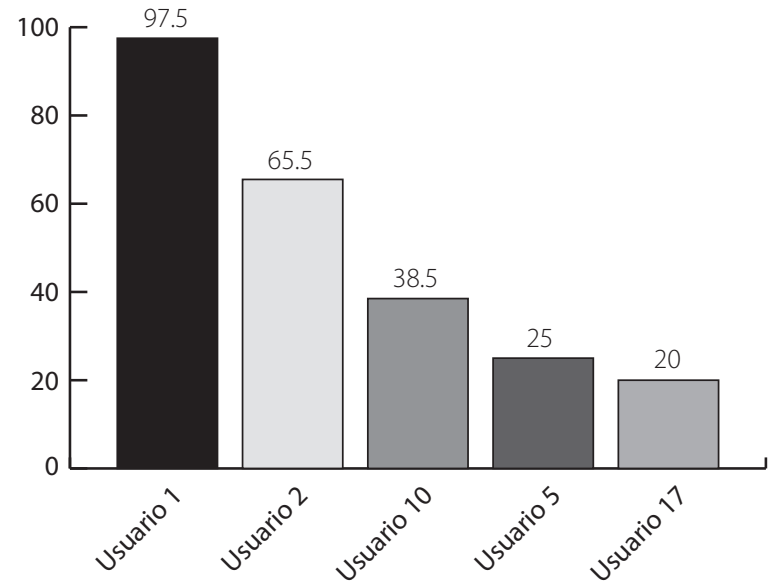

FIG. 8. Grado de intermediación de los usuarios en la red.

que motiva la participación en la Proto-Red, sin embargo esto no necesariamente genera procesos de formación en investigación. Si analizamos este resultado a la luz de los objetivos de Proto-Red, es posible observar entonces que efectivamente el primero de ellos se cumple, en tanto hay un flujo de información pero el mismo no genera colaboración o relaciones entre los centros, al menos no en el momento del estudio. Esta situación abre la posibilidad de un trabajo futuro más minucioso, que recurra a herramientas de naturaleza cualitativa para establecer si efectivamente el flujo de información refleja la colaboración y relación entre los centros participantes

Ahora bien, a nivel grupal los resultados muestran la existencia de nodos de la red, en este caso el grupo promotor de la red y los centros universitarios de Puriscal y Orotina, con mucha más actividad que otros, tanto a nivel de publicaciones como en la cantidad de visitas y comentarios que reciben. Este es un fenómeno esperable, ya que las redes sociales siempre cuentan con focos que concentran la actividad y que promueven el flujo de información en la misma (Gaete \& Vásquez 2008). Para efectos del futuro fortalecimiento de Proto-Red, el estudio en detalle y sistemático de la dinámica de estos centros focales puede brindar un conjunto de buenas prácticas a seguir para la inclusión de nuevos nodos a la red, particularmente desde una perspectiva que evalúe el tipo de relaciones y estrategias que le permite a estos nodos ser más productivos. Este punto es importante de resaltar porque según los supuestos del ARS (Wasserman \& Faust 1994), una red influye en las oportunidades y/o restricciones para la acción de sus integrantes, por ende es necesario evaluar más detalladamente qué propicia el éxito de un grupo y a su vez cuáles son las posibles limitantes con las que debe lidiar, y en consecuencia cuáles son las medidas a tomar para superarlas. 
Asimismo es de vital importancia considerar el producto de la sistematización de la participación de los usuarios. Se puede inferir que las categorías resultantes indican la presencia de comunidades virtuales incipientes alrededor de contenidos de interés para los miembros de la red ( $\mathrm{Li}$ et al. 2008). El hecho de que la cantidad de visitas a artículos relacionados con temas ecológicos y comunitarios sea alta a pesar de que no representan la mayoría de las publicaciones sugiere que efectivamente existen dichos intereses en la población que participa en Proto-Red (Müller 2004). Nuevamente este fenómeno puede tener una incidencia significativa en el devenir de Proto-Red. Si el énfasis de la dinámica de la red es comunitaria, entonces es posible pensar en potenciar los procesos desde dichas comunidades como una oportunidad para la formación en red y por ende para el desarrollo de habilidades en investigación. Un ejemplo que puede orientar a los promotores de la red es dado por Garrison \& Arbaugh (2007), quienes desarrollaron el marco de trabajo de Comunidades de Pesquisa, el cual impulsa comunidades educativas a distancia basadas en la colaboración. Si bien su énfasis no es la formación en investigación es una referencia indispensable en el contexto de la educación a distancia y por ende para Proto-Red.

A su vez el resultado anterior puede estar vinculado con la evidencia dada por las categorías de que la investigación no aparenta ser el tema de mayor interés para los usuarios de Proto-Red. Según la literatura es común que el uso de redes sociales virtuales para un propósito particular devenga en otro tipo de predilecciones por parte de sus usuarios, lo cual puede ciertamente afectar el propósito para el cual fue impulsada la red (Chow \& Chmura 2010). Al parecer los participantes de Proto-Red, si bien tienen interés en las actividades de la red, han buscado satisfacer sus necesidades en otros temas que en primera instancia no están vinculados con investigación. Este hecho sugiere que los promotores deben considerar nuevas estrategias para que Proto-Red cumpla con los objetivos establecidos inicialmente, por ejemplo propiciar nuevos procesos, donde la investigación sea una dinámica que intervenga y se involucre con el quehacer de las comunidades virtuales incipientes; los temas de interés evidenciados pueden ser objeto de estudios de caso guiados por los promotores $y$ por ende materia prima para procesos relacionados con la formación en red en habilidades para la investigación. Si Proto-Red desea consolidarse por antonomasia es necesario un modelo claro de formación en investigación adaptado a la dinámica que muestra la red, similar al elaborado por Willison (2009), donde el estudiante es guiado por una serie de niveles que encauzan su desarrollo y madurez como investigador. Claro está el marco de trabajo de dicho autor se circunscribe a un entorno de educación superior tradicional, lo cual representa una oportunidad para los promotores de Proto-Red de innovar mediante un modelo que responda a las particularidades de la educación a distancia.

Los resultados obtenidos de la visualización y evaluación mediante ARS de la red brindan una imagen de la estructura de la red, que denota la interacción de los usuarios en el momento del análisis. Este es un punto de partida para evaluar nuevamente en oportunidades posteriores la evolución de Proto-Red y dar seguimiento a su dinámica con un mayor nivel de detalle, con el fin de establecer algún tipo de causalidad que explique su cambio. Además el uso de ARS brinda una herramienta innovadora para la identificación cuantitativa de usuarios cuya participación en la red sobresale en relación a otros. El grado de intermediación es una métrica que permite distinguir usuarios que promueven y mantienen el flujo de información en Proto-Red (Guns et al. 2011). Los cinco usuarios con mayor puntaje en esta métrica pertenecen a centros universitarios que también se distinguen en cuanto a cantidad de publicaciones, comentarios y visitas se refiere, lo cual sugiere que estos usuarios han asumido un rol de promotor de la red en su propio contexto. Así, siguiendo el ejemplo de Guns et al. (2011), el grado de intermediación puede considerarse para la UNED como un indicador de usuarios con potencial en materia de investigación, los cuales una vez identificados pueden ser guiados en un proceso de formación en red basado en un modelo formal, tal y como se sugiere anteriormente.

Finalmente es necesario resaltar que Proto-Red es una red social cuya actividad plantea múltiples interrogantes en relación a la formación en red en la UNED, algunas de ellas ya han sido expuestas en este artículo, sin embargo el análisis desde otras perspectivas es indispensable para una comprensión más amplia del fenómeno. Se ha establecido que Proto-Red necesita de nuevas estrategias para su fortalecimiento y de un modelo de formación en red claro que le permita cumplir plenamente sus objetivos. Es importante además recalcar que la posibilidad de desarrollar indicadores desde el ARS, que colaboren en la identificación de usuarios y en la medición del grado de vinculación de estos con la red es un aporte innovador para la institución y plantea asimismo un reto para próximas investigaciones. Proto-Red es una iniciativa de experimentación ideal para que la UNED potencie procesos de formación en investigación innovadores basados en una dinámica en red, que puede motivar a otras instancias de la institución a considerar la formación en red como un elemento valioso para sus actividades. 


\section{AGRADECIMIENTOS}

Agradecemos el apoyo y financiamiento de la Vicerrectoría de Investigación de la UNED al proyecto "Análisis sociológico y computacional de la conformación de redes sociales en la Proto-Red de la Vicerrectoría de Investigación de la Universidad Estatal a Distancia y su impacto en el desarrollo de habilidades para la investigación" del cual surge esta publicación.

\section{REFERENCIAS}

AGNA Project. 2010. Applied Graph \& Network Analysis (http:// www.oocities.org/imbenta/agna/, consultado el 11 de agosto 2011)

Brandes, U., P. Kenis, \& J. Raab. 2005. La explicación a través de la visualización de redes. REDES: Revista Hispana para el análisis de redes sociales 9:1-18.

Cha, M., A. Mislove \& K.P. Gummadi. 2009. A measurement-driven analysis of informationpropagation in the Flickr Social Network. Proceedings of the 18th International World Wide Web Conference: 721-730.

Chinchilla, A.R, A. Segura, L. Bermúdez \& M. Barrientos. 2010. Informe, conclusiones y recomendaciones 2009: Proyecto Estratégico Proto-Red de Centros Universitarios para la investigación de la Vicerrectoría de Investigación de la UNED, Universidad Estatal a Distancia.

Chow, T. \& A. Chmura. 2010. Implementing a virtual community of interest at Capella University. SIGITE'10 - Proceedings of the 2010 ACM Conference on Information Technology Education:59-64.

Espuny, C., J. González, M. Lleixà \& M. Gisbert. 2011. Actitudes y expectativas del uso educativo de las redes sociales en los alumnos universitarios. Revista de Universidad y Sociedad del Conocimiento:171-185. (Disponible en http:// rusc.uoc.edu/ojs/index.php/rusc/article/view/v8n1espuny-gonzalez-Ileixa-gisbert/v8n1-espuny-gonzalezlleixa-gisbert, consultado el 26 de enero 2012).

Gaete, J.M. \& J.I. Vásquez. 2008. Conocimiento y estructura en la investigación académica: una aproximación desde el análisis de redes sociales. REDES: Revista Hispana para el análisis de redes sociales 14:1-34.

Garrison, D.R. \& J.B Arbaugh. 2007. Researching the community of Inquiry Framework: Review, Issues, and Future Directions. The Internet and Higher Education 10: 157-172.

Guns, R., Y.X. Liu \& D. Mahbuba. 2011. Q-measures and betweenness centrality in a collaboration network: A case study of the field of informetrics. Scientometrics 87: 133-147.
INEC. 2008. Sistema de consulta de Indicadores de uso y acceso de las TIC. (Disponible en www.inec.go.cr, consultado el 9 de setiembre del 2010).

Knoke, D. \& S. Yang. 2008. Social Network Analysis ser. Quantitative Applications in the Social Sciences 154, Sage, Londres, Londres, Inglaterra.

Lampe, C., D.Y.Wohn, J.Vitak, N.B. Ellison \& R. Wash. 2011. Student use of Facebook for organizing collaborative classroom activities. International Journal of Computer-Supported Collaborative Learning 6:329-347.

Li, X., L. Guo \& Y. Zhao. 2008. Tag-based social interest discovery. WWW'08: Proceeding of the 17th international conference on World Wide Web: 675-684.

Müller, C. 2004. Redes de "Comunidades personales" y "Comunidades de grupos" en diferentes servicios de comunicación en línea: Primeros resultados de un estudio empírico en Suiza. REDES: Revista Hispana para el análisis de redes sociales $5: 1-21$

Murtonen, M., E. Olkinuora, P. Tynjälä \& E. Lehtinen. 2008. Do I Need Research Skills in Working Life?: University Students' Motivation and Difficulties in Quantitative Methods Courses. The International Journal of Higher Education and Educational Planning 56: 599-612.

Nazir, A., S. Raza \& N. Chuah. 2008. Unveiling Facebook: A measurement study of social network based applications. Proceedings of 8th Internet Measurement Conference: 43-56.

Suárez, C. 2010. La formación en red como objeto de estudio, Revista de Universidad y Sociedad del Conocimiento 7:1-11. (Disponible en http://rusc.uoc.edu/ojs/index.php/rusc/ article/view/v7n2-suarez/v7n2-suarez; consultado el 26 de enero 2012).

Ulrike, P. \& Z. Panayiotis. 2009. Investigating social network patterns within an empathic online community for older people. Computers in Human Behavior 25:1139-1155.

UNED. 2009. Estatuto Orgánico, Centro de Información, Documentación y Recursos Bibliográficos (CIDREB). Universidad Estatal a Distancia, San Pedro, San José, Costa Rica.

Wasserman, S. \& K. Faust. 1994. Social Network Analysis: Methods and Applications. Cambridge University, Nueva York, Nueva York, Estados Unidos.

Wasserman, S., J. Scott \& P.J. Carrington. 2005. Introduction. p. 1-7 In Models and Methods in Social Network Analysis. Carrington, P.J, J. Scott. \& S. Wasserman. (Eds.). Cambridge University, Nueva York, Nueva York, Estados Unidos.

Willison, J. 2009. Multiple Contexts, Multiple Outcomes, One Conceptual Framework for Research Skill Development in Undergraduate Curriculum. CUR Quaterly 29:10-14. 